\title{
Signalling problem in absolutely unstable systems
}

Received: 11 May 2009 / Accepted: 1 September 2009 / Published online: 22 October 2009

\begin{abstract}
The dynamics of unstable systems crucially depends on the nature of the instability, either convective or absolute. The signalling problem, which is the study of the spatial response to a localized time-harmonic forcing, is generally believed to be relevant only for stable or convectively unstable systems and to be ill-posed for absolutely unstable systems, where the self-sustained perturbations grow faster than the forced harmonic response.

The present investigation shows that the signalling problem may still be well-posed for media displaying absolutely unstable regions. Considering weakly spatially inhomogenous systems, conditions are derived for the validity of the signalling problem. The complete spatial response to harmonic forcing is first analytically derived in terms of asymptotic approximations and then confirmed by direct numerical simulations.
\end{abstract}

Keywords absolute instabilities; causality; signalling problem; WKBJ

\section{Introduction}

In spatially homogenous systems, linear stability characteristics are derived from the dispersion relation $\omega=\Omega(k)$ between the frequencies $\omega$ and the wavenumbers $k$ of normal modes of the form $\mathrm{e}^{\mathrm{i}(k x-\omega t)}$, with $x$ and $t$ denoting streamwise distance and time respectively. These stability properties can be understood by resorting to different methods: temporal, spatial or spatio-temporal $[10,8,18]$.

In a temporal approach, a spatially harmonic perturbation of real wavenumber $k$ is considered. This wavelike initial perturbation evolves in time with a complex frequency $\omega$. Its spatial structure, determined by the wavenumber $k$, remains unchanged while its amplitude grows or decays with time. Growth or decay is determined by the sign of the temporal growth rate ${ }^{1} \omega_{i}$, while propagation takes place with a phase speed $\omega_{r} / k$. This analysis based on real wavenumbers and complex frequencies is known as the temporal problem.

In a spatial approach, localized harmonic forcing is applied with real frequency $\omega$, say at $x=0$. The spatial response to this forcing yields waves with complex wavenumbers $k$. The wavelength of the spatial response is determined by $k_{r}$ while the spatial growth or decay depends on $k_{i}$ : for $x \rightarrow+\infty$, the spatial response grows when $k_{i}<0$ and decays when $k_{i}>0$; the reverse holds for $x \rightarrow-\infty$. The analysis based on real frequencies and complex wavenumbers is known as the spatial, or signalling, problem.

\section{B. PIER}

Laboratoire de mécanique des fluides et d'acoustique,

École centrale de Lyon - CNRS - Université Claude-Bernard Lyon 1 - INSA Lyon, 36 avenue Guy-de-Collongue, 69134 Écully cedex, France.

1 Throughout this paper, subscripts $r$ and $i$ denote real and imaginary parts of complex values. 
The full spatio-temporal stability properties may be investigated by applying an impulsive localized perturbation: the analysis of the resulting wavepacket yields the complete dispersion relation between complex wavenumbers $k$ and complex frequencies $\omega$. While the impulsively started wavepacket decays in stable media, a growing response develops from the impulse location in unstable systems. If the growing wavepacket propagates away from its source and eventually leaves the medium unperturbed, the instability is said to be convective. If, by contrast, the instability grows in place and invades the system both upstream and downstream, the instability is said to be absolute. Convectively unstable (CU) systems do not display intrinsic dynamics and essentially behave as amplifiers: external perturbations are amplified while propagating through the system, and without continuous external input the medium returns to its unperturbed state. By contrast, absolutely unstable (AU) systems display nontrivial dynamics without external forcing: perturbations expand in both upstream and downstream directions so as to cover the entire domain and continue to grow exponentially at every point.

These stability concepts remain valid locally for spatially inhomogenous systems, provided the characteristic inhomogeneity length scale is large compared to a typical instability length scale. However, the connection between local stability characteristics and the long-term global dynamics of spatially developing systems is far from obvious. In a linear framework, it has been shown $[4,5,11]$ that the presence of local absolute instability is a necessary but not sufficient condition for global instability: in general an AU region of finite extent is required for a spatially developing medium to become globally unstable. Thus there exists a wide range of parameter settings for which a medium does not support any self-sustained fluctuations despite the presence of a region of local absolute instability. In such a situation, the linear signalling problem is legitimate and this is precisely the class of systems addressed in the present paper.

Globally stable but locally absolutely unstable systems are encountered in a variety of configurations of practical interest, among which wakes and boundary layers: the cylinder wake flow for Reynolds numbers in the range $25<\operatorname{Re}<49$ [12], a class of "synthetic" wake flows [16,17], the wake of a sphere [15], the three-dimensional boundary layer produced by a rotating disk [7].

The paper is organized as follows: After formulating the problem in terms of the widely used partial differential complex Ginzburg-Landau equation ( 2 ), its local ( $(3)$ and global ( $(4)$ stability properties are recalled. In $\S 5$, the correspondence between the complex space and frequency planes and the structure of the wavenumber branches are analysed. The complete analytic solution to the signalling problem is obtained in $\S 6$ in terms of asymptotic approximations and discussed in $\S 7$. These results are confirmed by direct numercial simulations in $\S 8$.

\section{Problem formulation}

Partial differential model equations account for the dynamics of a variety of physical systems [6] and are often tractable by analytical methods. The linearized complex Ginzburg-Landau model (1) has on many occasions proven to be a convenient testground to recognize and study generic features that have later been identified in a variety of situations. The same strategy is adopted here.

The system under consideration is assumed to be described by a complex scalar field $\psi(x, t)$ in an infinite one-dimensional spatially inhomogenous domain and it is governed by

$\frac{\partial \psi}{\partial t}=-\mathrm{i}\left(\omega_{0}(X)+\frac{1}{2} \omega_{k k}(X) k_{0}(X)^{2}\right) \psi+\omega_{k k}(X) k_{0}(X) \frac{\partial \psi}{\partial x}+\frac{\mathrm{i}}{2} \omega_{k k}(X) \frac{\partial^{2} \psi}{\partial x^{2}}+S(x, t)$,

where the complex functions $\omega_{0}(X), k_{0}(X)$ and $\omega_{k k}(X)$ account for the local properties of the medium and only depend on a slow space variable $X=\epsilon x$. The coefficients of (1) have been cast in this form for reasons that will become clear in the next section. The weak inhomogeneity parameter $\epsilon \ll 1$ is defined as the ratio of the typical instability length scale to the inhomogeneity length scale of the medium. The source function $S(x, t)$ represents an externally applied forcing to be specified below. While equation (1) applies to the real $x$-axis, the functions $\omega_{0}(X), k_{0}(X)$ and $\omega_{k k}(X)$ are assumed to be analytic and their continuation in the complex $X$-plane will be used in the following sections. 


\section{Local stability properties}

In the subsequent discussion, constant use is made of the local properties of system (1). Local characteristics are derived from (1) by freezing $X$ to some arbitrary (possibly complex) value and studying the corresponding spatially homogenous system

$\frac{\partial \psi}{\partial t}=-\mathrm{i}\left(\omega_{0}+\frac{1}{2} \omega_{k k} k_{0}^{2}\right) \psi+\omega_{k k} k_{0} \frac{\partial \psi}{\partial x}+\frac{\mathrm{i}}{2} \omega_{k k} \frac{\partial^{2} \psi}{\partial x^{2}}+S(x, t)$,

where the dependence of the coefficients on the parameter $X$ has been omitted. Normal modes of the form $\mathrm{e}^{\mathrm{i}(k x-\omega t)}$, with complex frequency $\omega$ and complex wavenumber $k$, are then governed by the local dispersion relation

$\omega=\Omega(k) \equiv \omega_{0}+\frac{1}{2} \omega_{k k}\left(k-k_{0}\right)^{2}$.

To satisfy causality, temporal growth rates must be bounded which requires that $\operatorname{Im} \omega_{k k}<0$. The particular form in which the coefficients of (1) and (2) have been cast brings to the fore the local complex absolute frequency $\omega_{0}$ associated with the local complex absolute wavenumber $k_{0}$ determined by the zero group velocity condition $[3,2]$

$$
\omega_{0}=\Omega\left(k_{0}\right) \quad \text { with } \quad \frac{\partial \Omega}{\partial k}\left(k_{0}\right)=0
$$

For impulsive forcing of the form $S(x, t)=\delta(x) \delta(t)$, where $\delta$ denotes the Dirac delta function, the absolute frequency $\omega_{0}$ characterizes the temporal evolution of the resulting wavepacket observed at a fixed station: when $\operatorname{Im} \omega_{0}<0$, the system is stable or convectively unstable and the wavepacket either decays or grows while being swept away by advection; when $\operatorname{Im} \omega_{0}>0$, the system is absolutely unstable and the impulse response exponentially grows at each point in space.

Now consider a spatially localized but temporally harmonic forcing of (possibly complex) frequency $\omega_{f}$, switched on at $t=0$, i.e., $S(x, t)=\delta(x) H(t) \mathrm{e}^{-\mathrm{i} \omega_{f} t}$ where $H$ denotes the Heaviside unit step function. The spatial response to this harmonic forcing consists in traveling waves of frequency $\omega_{f}$ and their wavenumbers are derived from (3) as

$k^{ \pm}\left(\omega_{f}\right)=k_{0} \pm \sqrt{2 \frac{\omega_{f}-\omega_{0}}{\omega_{k k}}}$.

Upon choosing the square-root branch cut in (4) along the positive real axis and with the square-root symbol denoting the root with positive imaginary part, the $k^{+}$and $k^{-}$branches pertain to the domains $x>0$ and $x<0$ on either side of the forcing. However, turning on the forcing at $t=0$ also produces a transient wavepacket that grows according to the absolute growth rate $\operatorname{Im} \omega_{0}$ at any fixed spatial location. Hence, two different situations arise depending on the relative values of $\operatorname{Im} \omega_{f}$ and $\operatorname{Im} \omega_{0}$ :

- When $\operatorname{Im}\left(\omega_{f}-\omega_{0}\right)>0$ the switch-on wavepacket is overwhelmed for large time by the spatial response tuned to the forcing frequency $\omega_{f}$, and the signalling problem is well-posed.

- When $\operatorname{Im}\left(\omega_{f}-\omega_{0}\right)<0$, the switch-on wavepacket overwhelms the spatial response at $\omega_{f}$ and the harmonic forcing does not succeed in tuning the medium to the externally applied frequency. The signalling problem is then ill-posed.

\section{Global stability properties}

In spatially inhomogenous systems governed by (1), the above discussion yields stability characteristics prevailing locally at each $X$. However the associated global behaviour cannot be immediately derived and must be analysed carefully $[11,5]$.

In a typical situation of interest, the local absolute growth rate $\operatorname{Im} \omega_{0}(X)$ displays a single maximum over the real $X$-axis and the medium is stable for $X \rightarrow \pm \infty$. In the same spirit as Huerre \& Monkewitz [9] and in order to keep computational difficulties to a minimum, it is assumed that

$\omega_{0}(X)=\omega_{s}+\frac{1}{2} \omega_{X X s}\left(X-X_{s}\right)^{2}$, 


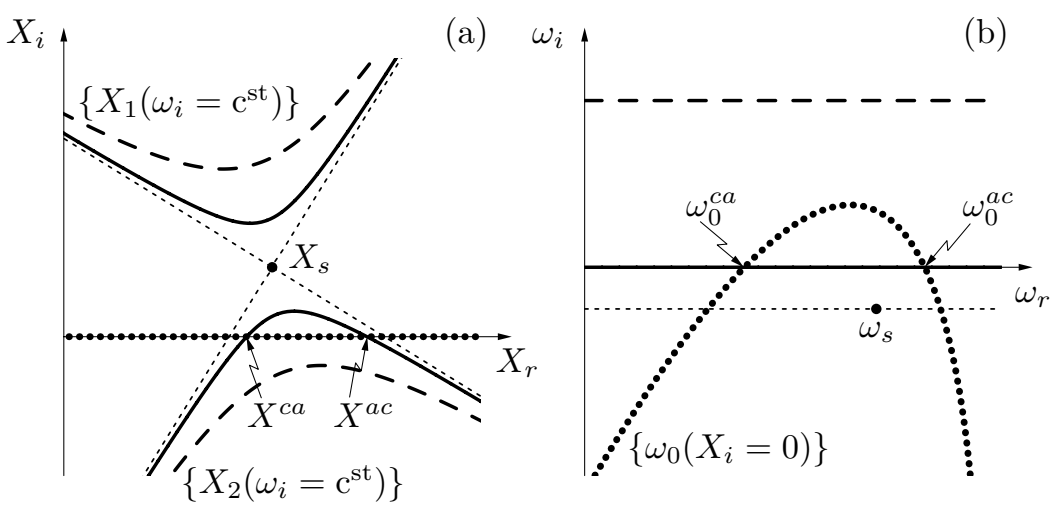

Fig. 1 Correspondence by the analytic mapping $\omega_{0}(X)$ between (a) complex $X$-plane and (b) complex $\omega$ plane. Associated curves are rendered by the same symbols. The real $X$-axis is mapped onto the dotted curve $\left\{\omega_{0}\left(X_{i}=0\right)\right\}$, and the real frequencies $\omega_{0}^{c a}, \omega_{0}^{a c}$ correspond to the boundaries $X^{c a}, X^{a c}$ of the AU interval. Horizontal lines in (b) are associated in (a) with the corresponding isolines $\left\{X_{1,2}\left(\omega_{i}=\mathrm{c}^{\mathrm{st}}\right)\right\}$ of constant absolute growth rate.

where $\omega_{s}, \omega_{X X s}$ and $X_{s}$ are complex parameters with $\operatorname{Im} \omega_{X X s}<0$. By resorting to asymptotic expansions, it has been shown $[11,5]$ that the impulse response of the spatially developing system is then dominated for large time by a global mode of frequency $\omega_{s}$. Hence, whenever $\operatorname{Im} \omega_{s}<0$ the system does not display self-sustained solutions: any perturbation eventually decays even though large amplification may be observed in the transient régime. Systems with $\operatorname{Im} \omega_{s}<0$ are thus globally stable whether or not they exhibit an interval of local absolute instability.

The paper is concerned with globally stable but locally absolutely unstable systems. In this situation all transient behaviour displays eventual decay, and it is legitimate to study the long-term spatial response to an externally applied time-harmonic forcing.

\section{Structure of complex $X$-plane and spatial branches}

When media are considered that are both globally stable and locally absolutely unstable, the local absolute frequency is of the form (5) with $\operatorname{Im} \omega_{s}<0$ and positive absolute growth rate $\left(\omega_{0, i}(X)>0\right)$ occurs over some finite interval $X^{c a}<X<X^{a c}$. Note that this is possible only if the saddle point $X_{s}$ of $\omega_{0}(X)$ is not located on the real $X$-axis. Let $\omega_{0}^{c a} \equiv \omega_{0}\left(X^{c a}\right)$ and $\omega_{0}^{a c} \equiv \omega_{0}\left(X^{a c}\right)$ denote the real marginal absolute frequencies at the boundaries of the AU interval.

The function $\omega_{0}(X)$ defines an analytic mapping between the complex $X$-plane and the complex $\omega$ plane as shown in figure 1 . The real $X$-axis is mapped onto the locus denoted as $\left\{\omega_{0}\left(X_{i}=0\right)\right\}$ (dotted parabolic curve in figure 1b) crossing the real frequency axis twice at $\omega_{0}^{c a}$ and $\omega_{0}^{a c}$, corresponding to the marginally absolutely unstable positions $X^{c a}$ and $X^{a c}$. Note that figure 1 illustrates a configuration where $\omega_{0}^{c a}<\omega_{0}^{a c}$ and $\operatorname{Im} X_{s}>0$; situations with $\omega_{0}^{a c}<\omega_{0}^{c a}$ and/or $\operatorname{Im} X_{s}<0$ yield similar pictures and results.

In the complex $X$-plane (figure 1a), isolines of constant absolute growth rate $\operatorname{Im} \omega_{0}(X)$ are obtained as preimages by $\omega_{0}^{-1}$ of horizontal lines in the $\omega$-plane. When such a line $\left\{\omega_{i}=\mathrm{c}^{\mathrm{st}}\right\}$ is located above the $\left\{\omega_{0}\left(X_{i}=0\right)\right\}$ curve in the $\omega$-plane (thick dashed line in figure $1 \mathrm{~b}$ ), the two corresponding isolines in the $X$-plane (thick dashed curves in figure 1a) do not cross the real axis and may be labeled $\left\{X_{1,2}\left(\omega_{i}=\mathrm{c}^{\mathrm{st}}\right)\right\}$, where the subscripts 1 or 2 correspond to curves confined to the upper or lower half $X$-planes respectively. When the line $\left\{\omega_{i}=\mathrm{c}^{\text {st }}\right\}$ is lowered onto the real $\omega$-axis, the associated $X_{1}$ and $X_{2}$ curves move towards each other. Since the real $\omega$-axis is crossed by the $\left\{\omega_{0}\left(X_{i}=0\right)\right\}$-curve at $\omega_{0}^{c a}$ and $\omega_{0}^{a c}$, one of the associated isolines (indicated by solid curves in figure 1a) necessarily crosses the real $X$-axis at $X^{c a}$ and $X^{a c}$. In figure $1(\mathrm{a})$, it is the lower $\left\{X_{2}\left(\omega_{i}=0\right)\right\}$-isoline that crosses the $X$-axis while the associated $\left\{X_{1}\left(\omega_{i}=0\right)\right\}$-isoline lies entirely within the upper half-plane. Note that the (thin-dashed) curves $\left\{X_{1,2}\left(\omega_{i}=\operatorname{Im} \omega_{s}\right)\right\}$ pinch at the saddle point $X_{s}$ for the line $\left\{\omega_{i}=\operatorname{Im} \omega_{s}\right\}$, which is however located in the lower frequency half-plane and will not be considered here. 
When solving the dispersion relation (3) with coefficients depending on $X$ for a given frequency $\omega_{f}$, the two spatial branches are obtained as

$k^{ \pm}\left(X, \omega_{f}\right)=k_{0}(X) \pm \sqrt{2 \frac{\omega_{f}-\omega_{0}(X)}{\omega_{k k}(X)}}$.

In the complex $X$-plane, these expressions display two branch points at $X_{1,2}\left(\omega_{f}\right)$, where $\omega_{0}(X)=\omega_{f}$, and branch cuts must be introduced. Upon choosing the branch cut for the square-root function in (6) along positive real values of its argument, the causality condition $\operatorname{Im} \omega_{k k}(X)<0$ guarantees that the branch cuts for $k^{ \pm}$lie within the regions where $\operatorname{Im}\left[\omega_{f}-\omega_{0}(X)\right]<0$ and thus do not intersect the region located between the $\left\{X_{1,2}\left(\omega_{i}=\operatorname{Im} \omega_{f}\right)\right\}$-curves where $\operatorname{Im}\left[\omega_{f}-\omega_{0}(X)\right]>0$. Hence the branch cut starting at $X_{1}\left(\omega_{f}\right)$ is located above the $\left\{X_{1}\left(\omega_{i}=\operatorname{Im} \omega_{f}\right)\right\}$-curve and the branch cut starting at $X_{2}\left(\omega_{f}\right)$ is located below the $\left\{X_{2}\left(\omega_{i}=\operatorname{Im} \omega_{f}\right)\right\}$-curve (see also figure 2 ). As indicated in $\S 3$, the spatial branches (6) may be unambiguously interpreted as downstream $k^{+}$or upstream $k^{-}$branches when $\operatorname{Im}\left[\omega_{f}-\omega_{0}(X)\right]>0$, which corresponds to the domain of the $X$-plane located between the $X_{1}$ and $X_{2}$ curves. The above choice of branch cuts thus extends the definition of the $k^{ \pm}\left(X, \omega_{f}\right)$ wavenumber branches to the entire complex $X$-plane for any frequency $\omega_{f}$ with $\operatorname{Im} \omega_{f}>\operatorname{Im} \omega_{s}$ (region above thindashed line in figure 1b). When considering real forcing frequencies $\omega_{f}$ in $(6)$, the choice of $k^{+}$and $k^{-}$branches corresponds to the downstream and upstream branches derived by traditional causality considerations in the stable or convectively unstable domain of the complex $X$-plane, characterized by $\operatorname{Im} \omega_{0}(X)<0$ and located between the solid $\left\{X_{1,2}\left(\omega_{i}=0\right)\right\}$-curves of figure 1 .

\section{Signalling problem in spatially developing medium}

For the signalling problem, the system is driven at some position on the real axis, say $X_{f}$, with a real frequency, say $\omega_{f}$, and the spatial response to this localized time-harmonic forcing is considered. Since the medium is assumed to be globally stable, any switch-on transients will eventually decay and it is legitimate to seek a long-term response of the form $\psi(x, t)=\phi(x) \mathrm{e}^{-\mathrm{i} \omega_{f} t}$, globally tuned to the forcing frequency.

Under the slowly-varying medium hypothesis characterized by $\epsilon \ll 1$, time-periodic solutions may be obtained in terms of WKBJ approximations [1] where the spatial structure is described by a rapidly varying complex phase, accounting for the local wavelength and spatial growth/decay rate, and a slowly varying envelope. For a given global frequency $\omega_{f}$, a WKBJ approximation is of the form

$\psi(x, t)=A(X) \exp \left[\frac{\mathrm{i}}{\epsilon} \int^{X} k\left(u, \omega_{f}\right) \mathrm{d} u-\mathrm{i} \omega_{f} t\right]$

where the envelope $A(X)$ is expanded in powers of $\epsilon$ as $A \sim A_{0}+\epsilon A_{1}+\ldots$ and governed by amplitude equations that can be computed recursively up to any order. In $(7)$, the local wavenumber $k\left(X, \omega_{f}\right)$ satisfies the local dispersion relation and follows one of the two spatial branches (6). Hence, in different domains of the complex $X$-plane, the solution may be approximated by different WKBJ expansions of the form (7) pertaining to different spatial wavenumber branches.

The above WKBJ expansions are singular [1] at each of the two turning points $X^{t}=X_{1,2}\left(\omega_{f}\right)$ of the dispersion relation, where $k^{+}\left(X^{t}, \omega_{f}\right)=k^{-}\left(X^{t}, \omega_{f}\right)$. These turning points are located on their respective $\left\{X_{1,2}\left(\omega_{i}=0\right)\right\}$-contours, see figure 2. From a turning point, three Stokes lines emerge, defined by $\operatorname{Im} \int_{X^{t}}^{X}\left[k^{+}\left(u, \omega_{f}\right)-k^{-}\left(u, \omega_{f}\right)\right] \mathrm{d} u=0$, and partition the complex plane into three different sectors. Along these Stokes lines both WKBJ approximations remain of the same order of magnitude, while inside the sectors one approximation is exponentially large with respect to the other.

Consider first the turning point $X_{1}^{t} \equiv X_{1}\left(\omega_{f}\right)$, located on the upper $\left\{X_{1}\left(\omega_{i}=0\right)\right\}$-contour in the complex $X$-plane (upper dashed curve in figure 2 ), and the two associated linearly independent WKBJ approximations

$\Psi_{1}^{ \pm}=A^{ \pm}(X) \exp \left[\frac{\mathrm{i}}{\epsilon} \int_{X_{1}^{t}}^{X} k^{ \pm}\left(u, \omega_{f}\right) \mathrm{d} u-\mathrm{i} \omega_{f} t\right]$.

Three Stokes lines and one branch cut emanate from $X_{1}^{t}$. The branch cut (dotted line), along which $k^{+}$ and $k^{-}$branches get exchanged, is located above the $\left\{X_{1}\left(\omega_{i}=0\right)\right\}$-curve and extends from $X_{1}^{t}$ towards 

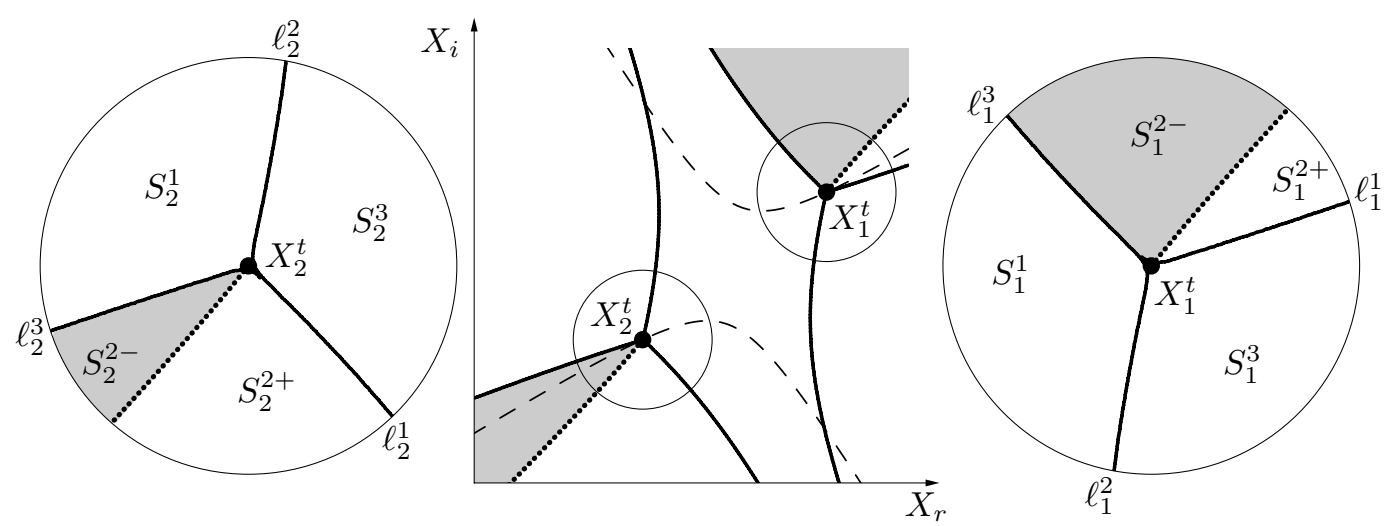

Fig. 2 Turning points and Stokes lines in the complex $X$-plane. The two turning points $X_{1,2}^{t}$ are located on their respective $\left\{X_{1,2}\left(\omega_{i}=0\right)\right\}$-isocontours (dashed lines). Each turning point is associated with a branch cut (dotted curves) and gives rise to a network of three Stokes lines (solid curves).

infinity in the upper complex $X$-plane. The three Stokes lines $\ell_{1}^{1}, \ell_{1}^{2}$ and $\ell_{1}^{3}$ divide the complex plane into three sectors $S_{1}^{1}, S_{1}^{2}$ and $S_{1}^{3}$, and sector $S_{1}^{2}$ is further divided into $S_{1}^{2-}$ and $S_{1}^{2+}$ by the branch cut, see zoom to right of figure 2. Along these Stokes lines both approximations $\Psi_{1}^{ \pm}$are of the same magnitude, and the dominant (resp. subdominant) solution becomes subdominant (resp. dominant) whenever a Stokes line is crossed.

Since the region near $X=+\infty$ is at most convectively unstable, the spatial response to the external forcing there necessarily features the $k^{+}$wavenumber branch and is thus made up of the subdominant $\Psi_{1}^{+}$approximation. Hence the dominant approximation $\Psi_{1}^{-}$is not present in the sector $S_{1}^{3}$, defined as the sector containing the region near $X=+\infty$, and the solution of the signalling problem in $S_{1}^{3}$ and along its bordering Stokes lines $\ell_{1}^{1}$ and $\ell_{1}^{2}$ is of the form $\psi \sim C_{1}^{+} \Psi_{1}^{+}$, solely made up of the subdominant $\Psi_{1}^{+}$approximation with, say, coefficient $C_{1}^{+}$. When continuing the solution from $S_{1}^{3}$ into $S_{1}^{1}$ across $\ell_{1}^{2}$, approximation $\Psi_{1}^{+}$becomes dominant. Inside sector $S_{1}^{1}$, any subdominant contribution of the form $C_{1}^{-} \Psi_{1}^{-}$may be present. On the next Stokes line $\ell_{1}^{3}$, both approximations are again of the same order, and the solution is then approximated by

$\psi \sim C_{1}^{-} \Psi_{1}^{-}+C_{1}^{+} \Psi_{1}^{+}$along $\ell_{1}^{3}$.

Similarly, when continuing the solution from $S_{1}^{3}$ into $S_{1}^{2+}$ across $\ell_{1}^{1}$, approximation $\Psi_{1}^{+}$becomes dominant and a solution of the form $C_{1}^{=} \Psi_{1}^{-}+C_{1}^{+} \Psi_{1}^{+}$must be considered inside sector $S_{1}^{2+}$. When crossing the branch cut, the approximations $\Psi_{1}^{+}$and $\Psi_{1}^{-}$get exchanged so that the same WKBJ superposition reads $C_{1}^{+} \Psi_{1}^{-}+C_{1}^{=} \Psi_{1}^{+}$in $S_{1}^{2-}$, with $\Psi_{1}^{-}$now the dominant term. On the Stokes line $\ell_{1}^{3}$, both approximations are again of the same order, and the solution is then approximated by

$\psi \sim C_{1}^{+} \Psi_{1}^{-}+C_{1}^{=} \Psi_{1}^{+}$along $\ell_{1}^{3}$.

Finally, comparing both expansions (9) and (10) shows that all coefficients $C_{1}^{=}, C_{1}^{-}$and $C_{1}^{+}$are identical to, say, $C_{1}$. In the different regions around turning point $X_{1}^{t}$, the following approximations to the signalling problem are then obtained

$\psi \sim \begin{cases}C_{1} \Psi_{1}^{+} & \text {in } S_{1}^{2+}, S_{1}^{3}, S_{1}^{1} \text { and along } \ell_{1}^{1}, \ell_{1}^{2}, \\ C_{1}\left(\Psi_{1}^{+}+\Psi_{1}^{-}\right) & \text {along } \ell_{1}^{3}, \\ C_{1} \Psi_{1}^{-} & \text {in } S_{1}^{2-} .\end{cases}$

Note that nothing special happens across the branch cut, except relabeling of the superscripts of $k^{ \pm}$ and $\Psi_{1}^{ \pm}$. Hence a result similar to the above holds even in situations where the orientation of the Stokes lines is such that the branch cut is not confined between $\ell_{1}^{1}$ and $\ell_{1}^{3}$ (see example in figure $3 \mathrm{a}$ ).

Thus it is only across the Stokes line $\ell_{1}^{3}$, defined as the Stokes line opposite the sector containing the region near $X=+\infty$ (see also figure 3 for further examples), that a change in the dominant wKBJ approximation occurs: a crossover between $k^{+}$and $k^{-}$as dominant wavenumber branches takes place across $\ell_{1}^{3}$ while everywhere else the dominant local wavenumber continuously depends on $X$. 
Consider now the turning point $X_{2}^{t} \equiv X_{2}\left(\omega_{f}\right)$, located on the lower $\left\{X_{2}\left(\omega_{i}=0\right)\right\}$-contour (lower dashed curve in figure 2). The same reasoning as above holds for the expansion of the spatial response in terms of the two associated WKBJ approximations

$\Psi_{2}^{ \pm}=A^{ \pm}(X) \exp \left[\frac{\mathrm{i}}{\epsilon} \int_{X_{2}^{t}}^{X} k^{ \pm}\left(u, \omega_{f}\right) \mathrm{d} u-\mathrm{i} \omega_{f} t\right]$.

The associated branch cut (dotted curve) is now located below the $\left\{X_{2}\left(\omega_{i}=0\right)\right\}$-curve. With Stokes lines and sectors around $X_{2}^{t}$ labeled as in figure 2 (left zoom), the solution to the signalling problem is then approximated by

$\psi \sim \begin{cases}C_{2} \Psi_{2}^{+} & \text {in } S_{2}^{2+}, S_{2}^{3}, S_{2}^{1} \text { and along } \ell_{2}^{1}, \ell_{2}^{2}, \\ C_{2}\left(\Psi_{2}^{+}+\Psi_{2}^{-}\right) & \text {along } \ell_{2}^{3}, \\ C_{2} \quad \Psi_{2}^{-} & \text {in } S_{2}^{2-},\end{cases}$

and crossover between the dominant $k^{+}$and $k^{-}$wavenumber branches takes place across the Stokes line $\ell_{2}^{3}$, located opposite the sector containing the region near $X=+\infty$. In the complex $X$-plane, the solution is thus dominated by the $k^{+}$branch everywhere except in the two sectors issuing from the turning points $X_{1}^{t}$ and $X_{2}^{t}$ respectively and delimited by the Stokes lines $\ell_{1}^{3}$ or $\ell_{2}^{3}$ and the corresponding branch cuts; these regions are indicated in grey in figure 2 . Note that, although the approximations (12) are formally identical to (8), the spatial branches in (12) are integrated from $X_{2}^{t}$ and the dominant/subdominant character depends on the behaviour for $X$ radiating away from this turning point.

The relationship between the constants $C_{1}$ and $C_{2}$ is readily derived by comparing the wKBJ expansions (11) and (13) in regions where they overlap, for example for $X \rightarrow+\infty$. From

$\begin{aligned} \psi & \sim C_{1} A^{+}(X) \exp \left[\frac{\mathrm{i}}{\epsilon} \int_{X_{1}^{t}}^{X} k^{+}\left(u, \omega_{f}\right) \mathrm{d} u-\mathrm{i} \omega_{f} t\right] \\ \text { and } \psi & \sim C_{2} A^{+}(X) \exp \left[\frac{\mathrm{i}}{\epsilon} \int_{X_{2}^{t}}^{X} k^{+}\left(u, \omega_{f}\right) \mathrm{d} u-\mathrm{i} \omega_{f} t\right]\end{aligned}$

it follows that

$C_{2}=C_{1} \exp \left[\frac{\mathrm{i}}{\epsilon} \int_{X_{1}^{t}}^{X_{2}^{t}} k^{+}\left(u, \omega_{f}\right) \mathrm{d} u-\mathrm{i} \omega_{f} t\right]$.

The above results $(11,13,14)$ entirely specify the asymptotic WKBJ approximation to the unique solution of frequency $\omega_{f}$ satisfying a causal boundary condition, i.e. made up of the $k^{+}$branch near $X=+\infty$. The spatial response of system (1) to harmonic forcing of frequency $\omega_{f}$ thus follows this solution over the real $X$-axis on the right side of the forcing location, for $X_{f}<X<+\infty$. When the external forcing is applied with $\mathcal{O}(1)$ amplitude, the response is also $\mathcal{O}(1)$ near $X_{f}$ which yields the magnitude of the remaining unknown constant as $C_{1} \sim \exp \frac{i}{\epsilon} \int_{X_{f}}^{X_{1}^{t}} k^{+}\left(u, \omega_{f}\right) \mathrm{d} u$. Note however that in this linear setting the precise value of $C_{1}$, and thus the exact spatial location of the forcing, does not influence the spatial structure of the response to the right side of the forcing.

The spatial response pertaining to the domain $-\infty<X<X_{f}$ on the left side of the forcing is similarly derived from the unique solution of frequency $\omega_{f}$ made up of the subdominant $k^{-}$branch for $X \rightarrow-\infty$. The corresponding details are straightforward and will not be presented here. In a typical situation of interest, forcing is applied upstream of the AU domain, i.e., $X_{f}<X^{c a}$, so that all relevant features concerning the structure of the spatial response over the AU interval are derived from the solution valid over $X_{f}<X<+\infty$.

\section{Discussion}

The previous analysis yields the spatial response to harmonic forcing over the entire $X$-axis, including the AU interval $X^{c a}<X<X^{a c}$.

For harmonic forcing of real frequency $\omega_{f}$ applied at some station $X_{f}$ upstream of the AU domain, local causality considerations suggest that the spatial response follows the $k^{+}\left(X, \omega_{f}\right)$ wavenumber 

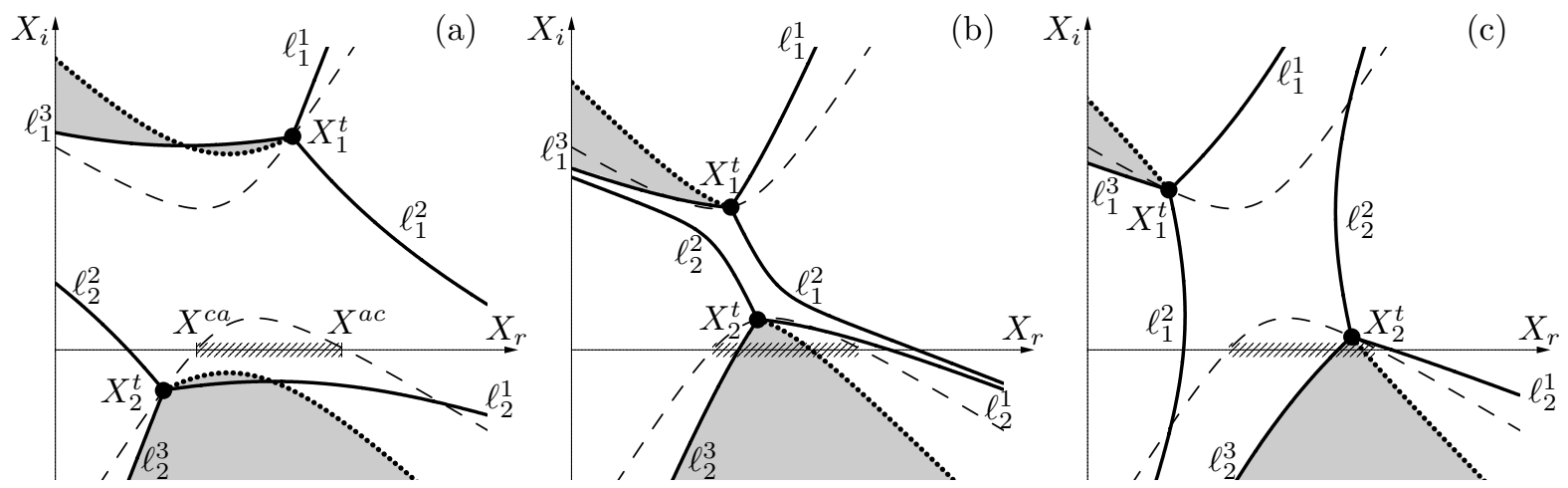

Fig. 3 Spatial structure of the signalling problem in the complex $X$-plane for different forcing frequencies $\omega_{f}$. The two turning points $X_{1,2}^{t}$ (thick dots) are located on their respective $\left\{X_{1,2}\left(\omega_{i}=0\right)\right\}$-isolines (dashed curves) and give rise to a network of Stokes lines (solid curves) and branch cuts (dotted lines). The spatial response is dominated by the $k^{-}$wavenumber branch in the shaded regions and by the $k^{+}$branch everywhere else. The AU interval of the real $X$-axis is hatched. (a) With $\omega_{f}$ outside the range $\omega_{0}^{c a}-\omega_{0}^{a c}$, the turning points $X_{1,2}^{t}$ are located in opposite half $X$-planes and the $k^{+}$wavenumber dominates over the entire real $X$-axis. (b,c) With $\omega_{f}$ within the range $\omega_{0}^{c a}-\omega_{0}^{a c}$, the turning points $X_{1,2}^{t}$ are located in same half $X$-planes, and the spatial response displays a jump in the dominant wavenumber on the real axis at the intersection with the $\ell_{2}^{3}$ Stokes line.

branch over the stable or $\mathrm{CU}$ interval $X_{f}<X<X^{c a}$, between the forcing location and onset of absolute instability. Similarly, in the at most convectively unstable downstream domain extending from $X=X^{a c}$ to $X \rightarrow+\infty$, the solution is obtained as the downstream response to some upstream located cause and is there also expected to follow the $k^{+}\left(X, \omega_{f}\right)$ wavenumber branch.

However, local considerations fail to predict the structure of the spatial response in the AU interval $X^{c a}<X<X^{a c}$ since the $k^{ \pm}$wavenumber branches (6) are there only formal solutions of the local dispersion relation and cannot be interpreted in terms of upstream or downstream spatial branches.

Based on the developments of the previous section, it will now be shown that in the AU region $X^{c a}<X<X^{a c}$ the structure of the spatial response and the dominant wavenumber essentially depend on the positions of the turning points $X_{1,2}^{t} \equiv X_{1,2}\left(\omega_{f}\right)$ relative to the real $X$-axis and thus on the relative values of $\omega_{f}, \omega_{0}^{c a}$ and $\omega_{0}^{a c}$.

Indeed, a forcing frequency $\omega_{f}$ outside the range $\omega_{0}^{c a}-\omega_{0}^{a c}$ lies above the $\left\{\omega_{0}\left(X_{i}=0\right)\right\}$ curve in the complex $\omega$-plane (dotted parabolic curve of figure 1b). The associated turning points are then located on either side of the real $X$-axis: $X_{1}^{t}$ in the upper half-plane and $X_{2}^{t}$ on parts of the $\left\{X_{2}\left(\omega_{i}=0\right)\right\}$ isoline in the lower half-plane, as illustrated in figure $3(\mathrm{a})$. In this situation, the branch cuts emanating from $X_{1,2}^{t}$ and respectively extending into the far upper and lower half $X$-planes do not cross the real $X$-axis. As a result, the spatial branches $k^{ \pm}\left(X, \omega_{f}\right)$ continuously depend on $X$ over the entire real axis, and the $k^{+}\left(X, \omega_{f}\right)$ branches prevailing on either side of the AU interval (hatched in figure 3 ) are continuously connected over the real $X$-axis. Note that the branch cut starting at $X_{2}^{t}$, while remaining below the $\left\{X_{2}\left(\omega_{i}=0\right)\right\}$-isoline, could cross the real axis twice before heading toward the far lower $X$-plane; however, the conclusions are not affected as long as an even number of crossings occurs since the branch cut could then be modified so as to avoid the $X$-axis. At the same time, the Stokes lines $\ell_{1}^{3}$ and $\ell_{2}^{3}$ over which an exchange of the dominant WKBJ approximations occurs do not cross the real $X$-axis either. The regions in the complex $X$-plane where the spatial response is dominated by the $k^{-}$ branch (shaded regions in figure 3a) then do not overlap the real $X$-axis. Hence the spatial response to harmonic forcing is approximated by the WKBJ approximation featuring the $k^{+}$wavenumber branch over the entire domain $X_{f}<X<+\infty$ including the AU interval $X^{c a}<X<X^{a c}$.

By contrast, for a forcing frequency $\omega_{f}$ within the range $\omega_{0}^{c a}-\omega_{0}^{a c}$, i.e. below the $\left\{\omega_{0}\left(X_{i}=0\right)\right\}$-curve in the complex $\omega$-plane, both turning points are located on the same side of the real $X$-axis: turning point $X_{2}^{t}$ has moved to the part of the $\left\{X_{2}\left(\omega_{i}=0\right)\right\}$-isoline located in the upper complex half-plane, as illustrated in figures $3(\mathrm{~b}, \mathrm{c})$. Thus the branch cut emanating from $X_{2}^{t}$ crosses the real $X$-axis and the spatial $k^{ \pm}\left(X, \omega_{f}\right)$ branches display a discontinuity across the branch cut. The $k^{+}\left(X, \omega_{f}\right)$ branches prevailing on either side of the AU interval are no longer continuously connected over the real $X$-axis. It follows that the local wavenumber of the spatial response to harmonic forcing applied at $X_{f}$ must 
display a jump somewhere in the AU interval $X^{c a}<X<X^{a c}$ if the $k^{+}$branches prevailing for both $X_{f}<X<X^{c a}$ and $X^{a c}<X<+\infty$ are to be reconciled. In this situation indeed, the real $X$-axis is also crossed by the $\ell_{2}^{3}$ Stokes line and, as shown in the previous section, an exchange in the dominant WKBJ solution approximating the spatial response precisely takes place across this Stokes line. Hence the region where the spatial response is dominated by the $k^{-}$branch (shaded regions in figures $3 \mathrm{~b}, \mathrm{c}$ ) overlaps the real $X$-axis, and along the AU interval both the branch cut and the $\ell_{2}^{3}$ Stokes line are encountered. As a result, the spatial response to harmonic forcing is then obtained in terms of both WKBJ approximations and the jump of the dominant local wavenumber occurs at the intersection of the $\ell_{2}^{3}$ Stokes line with the real $X$-axis.

\section{Confirmation by direct numerical simulation}

The structure of the spatial response derived by analytic methods in the previous sections has been confirmed by direct numerical simulations of system (1). The results presented in figure 4 have been obtained with a spatially varying local absolute frequency (5) defined by $\omega_{s}=2-0.5 \mathrm{i}, X_{s}=2+\mathrm{i}$ and $\omega_{X X s}=-1-2 \mathrm{i}$ and $\epsilon=0.1$, while the parameters $k_{0}=1-\mathrm{i}$ and $\omega_{k k}=1-0.5 \mathrm{i}$ were kept at constant values. These parameter settings correspond to an AU interval characterized by $X^{c a} \simeq 1.6, \omega_{0}^{c a} \simeq 3.2$ and $X^{a c}=3.3, \omega_{0}^{a c} \simeq-1.1$. Harmonic forcing is applied upstream at $X_{f}=1$ with frequencies $\omega_{f}=5$ (left sequence of figure 4) and $\omega_{f}=2$ (right sequence). The thick curves in figure 4 illustrate the different characteristics of the numerically computed spatial response: envelope $|\psi|$ and real part $\psi_{r}$ (first row), envelope on a logarithmic scale (second row), real part $k_{r}$ (third row) and imaginary part $k_{i}$ (fourth row) of local wavenumber. The thin curves in the wavenumber plots represent the wavenumber branches $k^{ \pm}\left(X, \omega_{f}\right)$ analytically computed via (6) with the corresponding $\omega_{f}$.

The forcing frequency $\omega_{f}=5$ lies outside the range $\omega_{0}^{c a}-\omega_{0}^{a c}$, so that the associated turning points $X_{1}^{t} \simeq 2.7+2.5 \mathrm{i}$ and $X_{2}^{t} \simeq 1.3-0.5 \mathrm{i}$ are located on either side of the real $X$-axis, which is thus free from any branch cuts. Figure $3(\mathrm{a})$ has been computed with exactly these parameter values. The critical Stokes lines $\ell_{1}^{3}$ and $\ell_{2}^{3}$ do not cross the real axis and the spatial response for $X>X_{f}$ is thus predicted to be entirely made up of the $k^{+}$wavenumber branch. Inspection of the numerical results given in figures 4(a1-a4) reveals that this is indeed the case. From figures 4(a1,a2) it is seen that the spatial response grows from $X_{f}$ through the entire AU interval to reach maximum amplitude beyond $X^{a c}$, before eventual decay further downstream. The local wavenumber in the spatial response, numerically computed as $-\mathrm{i} \partial_{x} \psi / \psi$ and corresponding to the thick curves in figures 4(a3,a4), very closely follows the analytically computed wavenumber branches (thin curves). The salient feature is that the spatial reponse follows the $k^{+}$branch in the entire domain to the right of the forcing location $X_{f}$, inluding the AU interval $X^{c a}<X<X^{a c}$ (delimited by dashed vertical lines).

The forcing frequency $\omega_{f}=2$ lies within the range $\omega_{0}^{c a}-\omega_{0}^{a c}$, so that the associated turning points $X_{1}^{t} \simeq 1.8+1.7 \mathrm{i}$ and $X_{2}^{t} \simeq 2.2+0.3 \mathrm{i}$ are both located in the upper complex half plane, as illustrated in figure 3(b) obtained with these parameter values. Now the Stokes line $\ell_{2}^{3}$ crosses the real axis for $X_{2}^{3} \simeq 1.9$ where a jump in the dominant wavenumber of the spatial reponse is predicted. This phenomenon is indeed observed in figures $4(\mathrm{~b} 3, \mathrm{~b} 4)$. The spatial response is seen to be dominated by the $k^{+}$branch from the forcing location $X_{f}$ to the Stokes line at $X_{2}^{3}$, beyond which the $k^{-}$branch dominates. This exchange in dominant WKBJ approximations at $X_{2}^{3}$ materializes by a cross-over of the numerically computed local wavenumber in figures $4(\mathrm{~b} 3, \mathrm{~b} 4)$ and by a kink of the envelope in figure 4(b2). The spatial response then follows the $k^{-}$branch from $X_{2}^{3}$ to the branch cut at $X_{\star} \simeq 3.0$ (vertical dotted line), beyond which the $k^{-}$branch is seemlessly relabelled as $k^{+}$. As anticipated from the previous section, the spatial response displays the $k^{+}$wavenumber branch in the two at most $\mathrm{CU}$ domains $X_{f}<X<X^{c a}$ and $X^{a c}<X<+\infty$. However, with $\omega_{f}$ in the range $\omega_{0}^{c a}-\omega_{0}^{a c}$ these branches are not continuously connected through the central AU interval. Hence the solution is there made up of both branches and cross-over of the dominant wavenumber occurs at the intersection with the Stokes line.

\section{Conclusion}

The signalling problem in spatially developing systems is fairly well understood for media that are at most convectively unstable, and it is generally believed to be ill-posed for absolutely unstable 

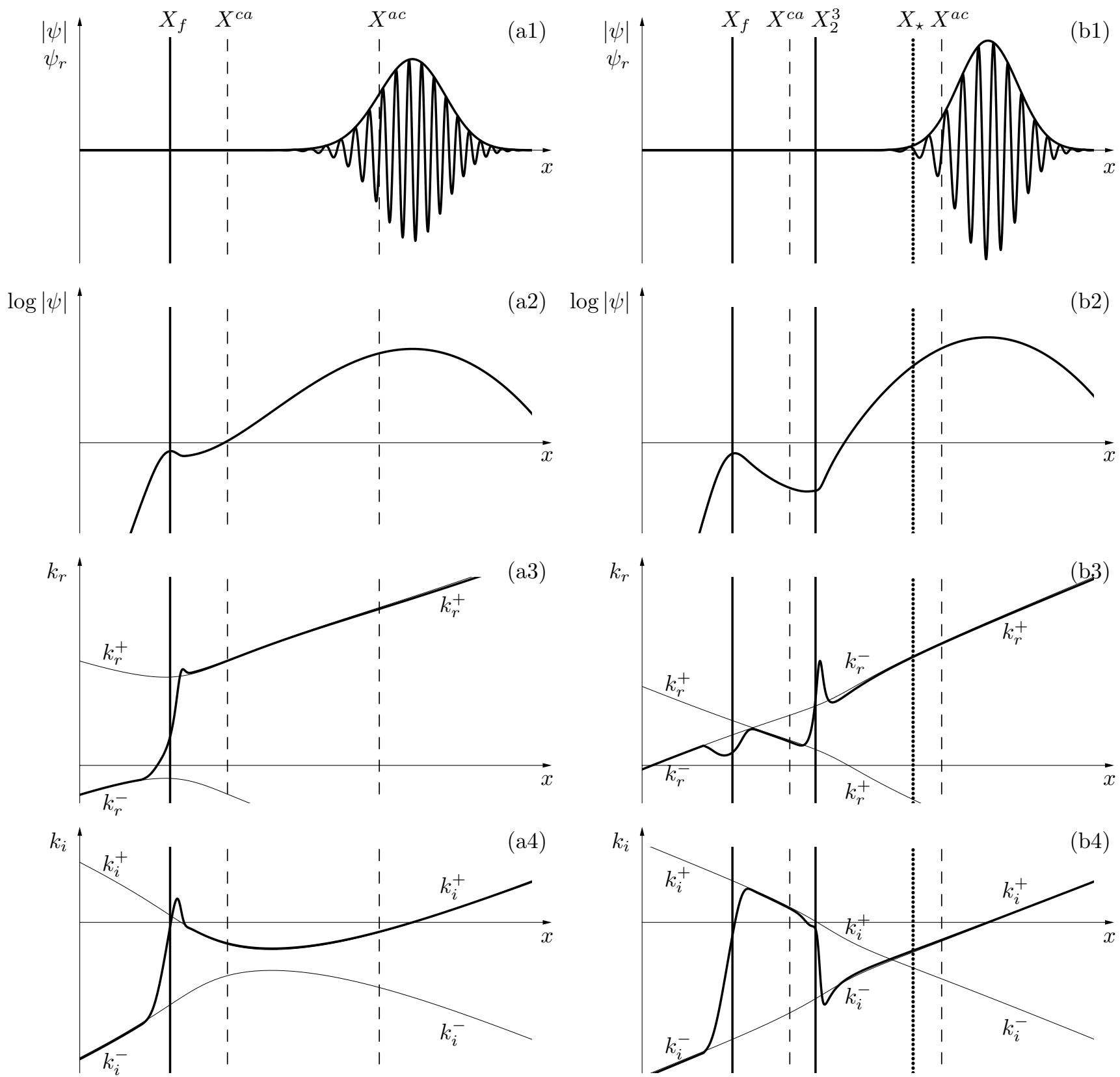

Fig. 4 Envelope $|\psi|$, real part $\psi_{r}$, local wavenumbers $k_{r}$ and $k_{i}$ of spatial response to harmonic forcing obtained by direct numerical simulation. Forcing is applied at $X_{f}$, upstream of the absolutely unstable interval $X^{c a}<X<X^{a c}$. (a) With $\omega_{f}$ outside the range $\omega_{0}^{c a}-\omega_{0}^{a c}$, the spatial response follows the $k^{+}$wavenumber branch for $X_{f}<X<+\infty$, including the AU interval. (b) For $\omega_{f}$ within the range $\omega_{0}^{c a}-\omega_{0}^{a c}$, the real $X$-axis intersects the Stokes line $\ell_{2}^{3}$ at $X_{2}^{3}$, where a jump in the dominant wavenumber is observed. Beyond the branch cut at $X_{\star}$, the $k^{-}$wavenumber branch prevailing for $X_{2}^{3}<X<X_{\star}$ becomes $k^{+}$and extends to $X=+\infty$. 
systems. The present investigation has shown that this problem remains well-posed for a certain class of absolutely unstable systems: whenever the system is globally stable (i.e. all global modes decay with time) all transient behaviour is eventually damped and a spatial response tuned at the forcing frequency establishes for large time. By resorting to asymptotic WKBJ expansions, the spatial structure of the time-harmonic response has been obtained in the entire domain, including the AU interval. In the AU interval, the dominant local wavenumber of the spatial response has been shown to depend on the positions in the complex $X$-plane of the turning points associated with the forcing frequency. The positions of these turning points depend on the relative values of the forcing frequency $\omega_{f}$ and the (real) marginal absolute frequencies $\omega_{0}^{c a}$ and $\omega_{0}^{a c}$ prevailing at the end points of the AU interval. The detailed analysis in the complex $X$-plane of the Stokes lines and branch cuts has revealed that two distinct situations arise:

- For forcing frequencies $\omega_{f}$ outside the range $\omega_{0}^{c a}-\omega_{0}^{a c}$, the local wavenumber observed in the spatial response continuously depends on streamwise distance and follows the $k^{+}$-branch everywhere downstream of the forcing location.

- For forcing frequencies within the range $\omega_{0}^{c a}-\omega_{0}^{a c}$, the spatial response displays a jump in the dominant local wavenumber branch at the location within the AU domain where the real $X$-axis is crossed by a Stokes line.

The above results have been analytically derived in a general setting and confirmed by numerical integration of the Ginzburg-Landau model equation. Work currently in progress concerns application of the present findings to the spatial response in the rotating-disk three-dimensional boundary-layer and its implications for an open-loop control strategy [13,14], based on localized harmonic forcing.

\section{References}

1. Bender, C.M., Orszag, S.A.: Advanced mathematical methods for scientists and engineers. McGraw-Hill, New York (1978)

2. Bers, A.: Space-time evolution of plasma instabilities — absolute and convective. In: M. Rosenbluth, R. Sagdeev (eds.) Handbook of plasma physics, pp. 451-517. North-Holland, Amsterdam (1983)

3. Briggs, R.J.: Electron-stream interaction with plasmas. M.I.T. Press, Cambridge, Mass. (1964)

4. Chomaz, J.M., Huerre, P., Redekopp, L.G.: Bifurcations to local and global modes in spatially developing flows. Phys. Rev. Lett. 60, 25-28 (1988)

5. Chomaz, J.M., Huerre, P., Redekopp, L.G.: A frequency selection criterion in spatially developing flows. Stud. Appl. Math. 84, 119-144 (1991)

6. Cross, M.C., Hohenberg, P.C.: Pattern formation outside of equilibrium. Rev. Mod. Phys. 65, 851-1112 (1993)

7. Davies, C., Carpenter, P.W.: Global behaviour corresponding to the absolute instability of the rotating-disk boundary layer. J. Fluid Mech. 486, 287-329 (2003)

8. Huerre, P.: Open shear flow instabilities. In: G.K. Batchelor, H.K. Moffatt, M.G. Worster (eds.) Perspectives in Fluid Dynamics, pp. 159-229. Cambridge University Press, Cambridge (2000)

9. Huerre, P., Monkewitz, P.A.: Local and global instabilities in spatially developing flows. Ann. Rev. Fluid Mech. 22, 473-537 (1990)

10. Huerre, P., Rossi, M.: Hydrodynamic instabilities in open flows. In: C. Godrèche, P. Manneville (eds.) Hydrodynamics and Nonlinear instabilities, pp. 81-294. Cambridge University Press (1998)

11. Le Dizès, S., Huerre, P., Chomaz, J.M., Monkewitz, P.A.: Linear global modes in spatially developing media. Phil. Trans. R. Soc. Lond. A 354, 169-212 (1996)

12. Monkewitz, P.A.: The absolute and convective nature of instability in two-dimensional wakes at low Reynolds numbers. Phys. Fluids 31, 999-1006 (1988)

13. Pier, B.: Open-loop control of absolutely unstable domains. Proc. R. Soc. Lond. A 459, 1105-1115 (2003)

14. Pier, B.: Primary crossflow vortices, secondary absolute instabilities and their control in the rotating-disk boundary layer. J. Eng. Math. 57, 237-251 (2007)

15. Pier, B.: Local and global instabilities in the wake of a sphere. J. Fluid Mech. 603, 39-61 (2008)

16. Pier, B., Huerre, P.: Nonlinear self-sustained structures and fronts in spatially developing wake flows. J. Fluid Mech. 435, 145-174 (2001)

17. Pier, B., Huerre, P.: Nonlinear synchronization in open flows. J. Fluids Struct. 15, 471-480 (2001)

18. Schmid, P.J., Henningson, D.S.: Stability and transition in shear flows. Applied Mathematical Sciences. Springer, New York (2001) 